\title{
Relação entre atividade física e sobrecarga mecânica na articulação gleno-umeral
}

\author{
Antonio Pakenas \\ Júlio C. Serrão
}

\author{
Laboratório de Biomecânica \\ Escola de Educação Física e Esporte \\ Universidade de São Paulo \\ Brasil
}

\section{RESUMO}

A articulação gleno-umeral (AG) é caracterizada pela maior liberdade de movimento no corpo humano. A principal proposta desta revisão foi discutir, a partir da análise das características anatômicas e funcionais da AG, aspectos relacionados à sobrecarga mecânica em algumas de suas estruturas. A literatura especializada evidencia que a sobrecarga mecânica imposta às estruturas da AG é condicionada por diversos fatores, dentre os quais as características da atividade física realizada. Outros fatores que não podem ser desprezados incluem as variações anatômicas das estruturas gleno-umerais, a faixa etária do indivíduo e o grau de afinidade do indivíduo com a atividade física.

Palavras-chave: articulação gleno-umeral, sobrecarga, esporte.

\begin{abstract}
Relationship between physical activity and mechanical overload in the glenohumeral joint

The glenohumeral joint (GJ)) is characterised by having the greatest freedom of movement of the human body. The primary purpose of this review was to discuss, based on analysis of the anatomical and functional characteristics of the GJ, aspects in relation to mechanical overload in the joint. The specialised literature shows that mechanical overload applied to GJ structures depend of several factors - physical activity is among these factors. Other conditions that should not be overlooked include anatomical variations of the GJ structures, age of the individual, and skill level of the individual with reference to physical activity.
\end{abstract}

Keywords: glenohumeral joint, overload, sport. 


\section{INTRODUÇÃO}

A articulação gleno-umeral (AG) é caracterizada pela maior liberdade de movimento no corpo humano. A mobilidade é possibilitada pela forma côncavo-convexa dos ossos que se articulam (28). Desse modo, a AG é a articulação que mais depende dos mecanismos de estabilização, a fim de manter sua mobilidade com o risco mínimo de lesão. As estruturas estabilizadoras, que podem ser passivas ou ativas, incluem, no primeiro caso, a cápsula articular, seus ligamentos e o lábio glenóide. Constituem as estruturas ativas o grupo muscular denominado manguito rotador: subescapular, redondo menor, supra-espinhoso e infra-espinoso. Os movimentos do úmero são principalmente gerados por quatro músculos agonistas: deltóide, peitoral maior, grande dorsal e redondo maior. Todavia, os manguitos também são responsáveis pelo movimento do úmero, assim como os músculos motores podem agir como estabilizadores em diversas ocasiões. O equilíbrio de forças entre as estruturas estabilizadoras passivas e ativas e os músculos motores do úmero, além dos músculos que movem a escápula, possibilita a estabilização da cabeça umeral na cavidade glenóide (43).

Entretanto, o grau de comprometimento dessas estruturas em relação à estabilização e mobilidade varia com a posição da AG, e também de acordo com a atividade física realizada (38).

\section{OBJETIVOS}

A principal proposta desta revisão foi discutir, a partir da análise das características anatômicas e funcionais da AG, aspectos relacionados à sobrecarga mecânica imposta as suas estruturas, em função das características dos movimentos realizados.

\section{SOBRECARGA NA CÁPSULA ARTICULAR E LIGAMENTOS}

A cápsula articular e seus ligamentos estão sujeitos a lesões que são relativamente comuns durante a execução de alguns movimentos, como por exemplo aqueles que envolvem rotação externa da AG (16). Já que há um consenso de que os ligamentos pelo menos evitam a relação anormal entre os ossos de uma articulação em situações estáticas, além de servirem como órgãos de propriocepção (33), um tópico sobre a cápsula e seus ligamentos é necessário. Em geral, os livros de anatomia básica descrevem a cápsula gleno-umeral contendo três ligamentos: liga- mento gleno-umeral superior (LGS), ligamento gleno-umeral médio (LGM) e ligamento gleno-umeral inferior (LGI) (3). Todavia, estudos envolvendo dissecação de cadáveres mostram que a área de seção transversa e a organização das fibras colágenas (ou mesmo a presença dos ligamentos superior e médio) não é constante, o que levou os pesquisadores que conduziram esses estudos a crer que essas estruturas podem não ter grande significado funcional $(23,45)$. Outros pesquisadores têm proposto mecanismos alternativos para a prevenção da subluxação inferior do úmero (função do LGS e LGM), como por exemplo, a pressão intra-articular negativa e o tônus muscular (18).

Contudo, experimentos em que os ligamentos da cápsula articular gleno-umeral são testados em relação à deformação exibida em determinadas posições mostram que não somente o LGI, como também o LGM e o LGS, são importantes para evitar a subluxação do úmero $(24,41)$. Nesses experimentos, os ombros dos cadáveres são colocados num dispositivo que possibilita a fixação da escápula e a movimentação do úmero, sendo que a articulação pode ser abduzida em variados graus e também rodada, enquanto medidores de deformação inseridos nos ligamentos registram as alterações na solicitação mecânica imposta aos ligamentos, conforme a posição alcançada. Os resultados evidenciam que, à medida que o úmero é abduzido, diminui a solicitação sobre o LGS, aumentando a solicitação sobre o LGM e o LGI (principalmente sobre o último). Além disso, quando a AG é levada até uma posição de $45^{\circ}$ ou $90^{\circ}$ de abdução e então rodada externamente, mais solicitação é colocada sobre o LGI. Porém, como apenas o úmero era movido nesses experimentos e a contribuição da rotação superior da escápula para esse movimento é significativa (31), a observada solicitação refere-se preferivelmente a graus de abdução maiores que $45^{\circ}$ e $90^{\circ}$.

Os resultados das pesquisas anteriores para o LGI parecem encontrar respaldo quando estudos de caso são analisados. Exercícios com pesos, que colocam a AG na posição de abdução e máxima rotação externa, tem sido relacionados como causa de instabilidade anterior da AG (11). A modificação da técnica usada nos exercícios, isto é, a prevenção da rotação externa máxima, parece ser suficiente para sanar o problema. 


\section{SOBRECARGA NOS MANGUITOS ROTADORES E MÚSCULOS MOTORES}

Os manguitos rotadores, assim como a cápsula articular e seus ligamentos, devem resistir a diferentes sobrecargas, dependendo da posição da AG. O músculo supra-espinhoso parece ser mais susceptível às lesões quando a AG está na posição aduzida, já que nesse caso uma área do seu tendão de inserção, denominada zona crítica, torna-se hipovascularizada (34). Embora o envelhecimento do indivíduo seja reconhecido como um fator que agrava essa zona crítica, tanto em extensão da área atingida como também em maior hipovascularidade $(46,25,20)$, nenhuma evidência aponta de maneira decisiva a existência de relação entre a atividade física, neste caso o hábito de carregar objetos com auxílio da AG aduzida $_{\llcorner}$e a aceleração desse processo. Além disso, se o fato da cavidade glenóide estar rodada na direção superior (2), na vertical ou inferiormente rodada (7), influencia esse envelhecimento, nenhum desses pesquisadores pôde fornecer evidências. Desse modo, fica-se na dependência de estudos futuros que tragam novas evidências sobre essa importante questão. O músculo supra-espinhoso também tem sido implicado no mecanismo que evita a subluxação superior da cabeça umeral durante os movimentos de abdução e flexão da AG, o que poderia causar o impingimento de pelo menos três tendões (supra e infra-espinhoso, além da cabeça longa do bíceps braquial) contra o terço anterior do acrômio (21). Entretanto, não existem evidências suficientes para sustentar que de fato, esse é o principal responsável pela estabilização da cabeça umeral na cavidade glenóide, haja vista que os experimentos que utilizaram o bloqueio dos potenciais de ação dos nervos supra-escapular e axilar (44, 13) não contaram, simultaneamente, com nenhuma análise de imagem da articulação.

Quanto ao subescapular, pesquisadores tem evidenciado que esse músculo está propenso a lesões quando age como estabilizador da AG durante os movimentos de abdução e rotação externa da articulação, evitando a subluxação anterior do úmero. Isso foi demonstrado em experimentos que contaram com a utilização de cadáveres, já que o corte intencional do tendão de origem do músculo subescapular possibilitou o deslocamento anterior do úmero (40). Outros estudos tem mostrado que, entre indivíduos que passaram por um procedimento cirúrgico conhecido como Putt-Platt (imbricação da cápsula e músculo subescapular), somente tiveram recidivas aqueles que exibiram um aumento no grau de rotação externa da AG durante a reavaliação (12). Além disso, há ainda a evidência trazida por um estudo (8) que documentou 16 casos de ruptura parcial ou total do tendão do músculo subescapular. A maioria dos pacientes experimentou essa situação após um episódio de violenta rotação externa da AG com o braço aduzido (sete pacientes) ou violenta extensão (seis pacientes), enquanto o restante sofreu acidentes envolvendo queda. Os sinais observáveis foram a aumentada rotação externa e a diminuição na geração de força em direção à rotação interna.

Curiosamente, nenhum sinal de instabilidade foi observado nos sujeitos, reforçando a hipótese que o músculo subescapular, e não a cápsula articular, é a principal estrutura envolvida na restrição da rotação externa da AG. Contudo, devemos ressalvar que os testes realizados por esses dois autores servem para diagnosticar preferivelmente frouxidão ligamentar, porém não instabilidade, já que a articulação não foi testada em condições dinâmicas (5).

Em relação ao músculo infra-espinhoso, não há de fato uma posição da AG que o torne potencialmente vulnerável às lesões. Embora o tendão de inserção do músculo infra-espinhoso também apresente uma zona crítica como aquela existente no tendão do músculo supra-espinhoso, essa não é tão severa e extensa. A única estrutura relacionada ao músculo infra-espinhoso que parece sofrer lesões de forma freqüente é o ramo do nervo supra-escapular que segue para o músculo infra-espinhoso. As lesões observadas geralmente ocorrem no local denominado como incisura espinoglenoidal (27). As atividades físicas que exigem do praticante a execução de arremessos ou saques cuja fase de desaceleração é breve, como o saque tático do voleibol, parecem ser as prováveis causadoras do problema (6), que também pode afetar indivíduos que praticam o voleibol de maneira recreativa (1). Todavia, deve-se apontar que a relação entre o curto tempo de duração da fase de desaceleração, que exigiria uma contração muscular excêntrica mais vigorosa, e a lesão é apenas circunstancial._ Em relação aos músculos denominados agonistas, o peitoral maior merece destaque, pois é uma estrutu- 
ra que, embora aparentemente robusta, possui relativa fragilidade, haja vista os estudos de caso sobre rupturas do seu tendão de inserção durante o movimento de adução horizontal da AG, por exemplo na execução do supino, um exercício comum em programas de condicionamento físico(17). Além disso, os estudos em que a eletromiografia foi utilizada, evidenciam acentuada atividade desse músculo durante a execução dos clássicos exercícios de flexão de braço e (principalmente) flexão de braço com as mãos separadas (42). Esses exercícios devem, então, ser ministrados com cautela, de forma a não causar qualquer tipo de lesão.

\section{SOBRECARGA DURANTE A EXECUÇÃO DE HABILIDADES MOTORAS}

As atividades esportivas abordadas neste tópico incluirão a natação (técnica crol), o tênis e o arremesso (especificamente o baseball), pois são as atividades relacionadas a lesões da articulação glenoumeral mais estudadas. Os dados são colocados de forma qualitativa, haja vista que a metodologia usada em trabalhos preliminares não foi mantida em trabalhos subsequentes, impossibilitando a comparação entre os dados de maneira quantitativa.

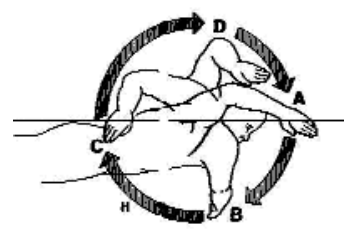

Figura 1: Fases da natação (técnica crol): propulsão inicial $(A B]$, propulsão final $[B C]$, recuperação inicial $(C D)$ e recuperação final (DA). (Adaptado de PINK et al., 1991).

Em relação à natação (técnica crol), alguns pesquisadores optam por dividi-la em quatro fases: recuperação inicial, recuperação final, propulsão inicial e propulsão final (35), como mostra a Figura 1. Os músculos mais solicitados nessa técnica (pelo menos para nadadores em competições universitária e master) são o subescapular, supra-espinhoso e redondo menor, já que estão moderadamente ativos em mais de $50 \%$ do ciclo do nado (30). O músculo subescapular parece ser o mais solicitado, pois suas vigorosas contrações impediram até mesmo o registro de sua atividade (22), haja vista o deslocamento significativo dos eletrodos implantados no músculo.

Os estudos sobre o arremessar tem sido realizados principalmente em relação ao baseball $(9,10,14,15)$ e ao tênis (37). O arremesso e o saque no tênis tem sido divididos em quatro fases (Figura 2), a saber: 1) preparação, bastante variável entre os sujeitos e caracterizada por uma ativação muscular pequena; 2) cocking inicial e final, todavia, analisaremos apenas a última, caracterizada pela máxima rotação externa da AG, devido a sua maior importância; 3) aceleração, que começa após a fase cocking final e termina exatamente no lançamento da bola ou impacto da raquete sobre a bola; 4) desaceleração, que começa após a fase de aceleração e termina quando o membro é refreado $(26,37)$.

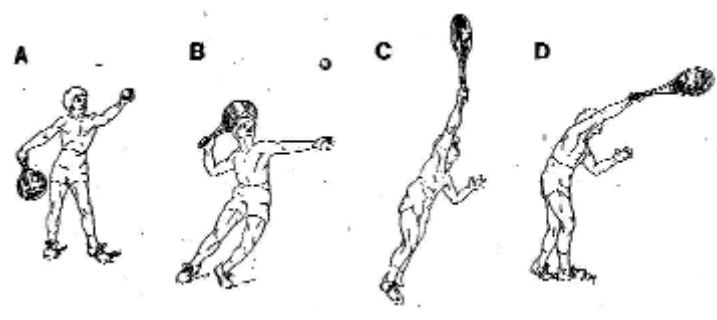

Figura 2: Fases do saque: wind-up $[A]$, cocking $(B]$, aceleração $[C]$ e desaceleração (D). (Adaptado de RYU et al., 1988).

Em relação ao arremesso no baseball, durante a fase cocking os músculos que estão mais ativos são o subescapular (sem dúvida o mais importante nessa fase, porque restringe a rotação externa da AG, embora também seja auxiliado pelo peitoral maior nessa função), infra-espinhoso e redondo menor (estes responsáveis pela rotação externa da AG). Durante a fase de aceleração, os músculos mais ativos são o subescapular, o peitoral maior e o grande dorsal, pois fazem com que o braço seja lançado à frente. Já na fase de desaceleração, são os músculos 
infra-espinhoso e redondo menor os mais ativos, pois controlam a adução e rotação interna da AG (9, $10,15)$. Porém, esse é o padrão mostrado por jogadores profissionais. Indivíduos que jogaram há anos em campeonatos amadores e apenas de forma recreativa no presente, ou jogadores profissionais com instabilidade anterior, exibem padrões distintos de ativação muscular. A característica mais importante no padrão desses grupos de indivíduos é o uso acentuado do mecanismo de estabilização, que oferece maior segurança, porém menor velocidade durante o arremesso. Dessa forma, os músculos supraespinhoso e bíceps braquial encontram-se bastante ativos, além de maior ativação dos músculos que restringem o movimento em cada uma das fases do arremesso, ao mesmo tempo em que ocorre diminuição da atividade dos músculos responsáveis pela aceleração do membro $(9,14)$.

O padrão de ativação muscular do saque no tênis é semelhante, embora possua algumas peculiaridades, ao do arremesso. Num estudo com jogadores de tênis amadores, pesquisadores (37) observaram que o músculo infra-espinhoso está ativo em todas as três fases do saque, o subescapular está mais ativo nas fases cocking e aceleração, enquanto o supra-espinhoso se encontra mais ativo nas fases cocking e desaceleração. Em relação aos músculos peitoral maior e grande dorsal, ambos estão bastante ativos na fase de aceleração.

Alguns autores $(4,19)$ tem defendido que, nas atividades em que o braço realiza movimentos de arremessar, braçadas e saques, há ênfase em relação aos movimentos de adução e rotação interna. Isso causaria um desequilíbrio ainda maior do que o normalmente existente (39) na capacidade de geração de força entre os rotadores internos e externos e entre os adutores e abdutores, com conseqüente risco aumentado (hipotético) de lesão no ombro. O risco seria iminente se houvesse instabilidade da articulação glenoumeral. De fato, seus experimentos utilizando dinamômetros isocinéticos mostram que a razão entre o momento gerado pelos adutores/ rotadores internos e abdutores/ rotadores externos é maior em atletas (tenistas e nadadores) do que em não são atletas. Todavia, devemos interpretar esses resultados com cautela, pois o uso da escala de razão em experimentos que empregam os dinamômetros isocinéticos é muitas vezes inadequado (36).

\section{CONCLUSÃO}

A literatura especializada evidencia que as sobrecargas mecânicas aplicadas às estruturas da AG dependem de vários fatores, dentre os quais a atividade física realizada. Outros fatores que não podem ser desprezados incluem as variações anatômicas das estruturas gleno-umerais, a faixa etária do indivíduo e o grau de afinidade que o indivíduo mostra em relação à atividade física. Logo, diversas pessoas que praticam uma determinada atividade com freqüência e intensidade semelhantes podem sofrer conseqüências diferentes. Desse modo, a promoção de um aumento na resistência das estruturas mais sobrecarregadas, através de programas de fortalecimento muscular planejados especificamente para cada atividade, pode ser uma proposta razoável para o praticante de atividade física $(29,32)$. Apesar de alguns autores defenderem a tese de que há um desequilíbrio muscular entre músculos estabilizadores e agonistas, devemos lembrar que essas funções podem ser permutadas, isto é, músculos agonistas podem agir como estabilizadores e vice-versa. Desse modo, recomenda-se cautela na interpretação do que é geralmente designado equilíbrio muscular.

\author{
CORRESPONDÊNCIA \\ Júlio C. Serrão \\ Laboratório de Biomecânica \\ Escola de Educação Física e Esporte \\ Universidade de São Paulo \\ Av. Prof. Melo de Moraes, 65 \\ CEP 05508-900, Cidade Universitária \\ São Paulo, SP \\ Brasil \\ Fone/fax: (11) 30913184 \\ jcserrao@usp.br
}




\section{REFERÊNCIAS BIBLIOGRÁFICAS}

1. Andrade, RP, Pires PR, Pereira JAR (1993). Compressão do nervo supra-escapular: avaliação de sete casos. Revista Brasileira de Ortopedia, v.28, n.9, p.645-9.

2. Basmajian JC, Bazant FJ (1959). Factors preventing downward dislocation of the adducted shoulder joint. Journal of Bone and Joint Surgery [Am], v.41, n.7, p.1182-6.

3. Castro SV (1973). Anatomia fundamental. McGraw-Hill, Rio de Janeiro.

4. Chandler TJ, Kibler WB, Stracener EC, Ziegler AK, Pace B (1992). Shoulder strength, power, and endurance in college tennis players. The American Journal of Sports Medicine, v.20, n.4, p.455-8.

5. Clancy Jr WG (1983). Knee ligaments injury in sports: the past, present and future. Medicine and Science in Sports and Exercise, v. 15, n.1, p.9-14

6. Ferreti A, De Carli A, Fontana M (1998). Injury of the suprascapular nerve at the spinoglenoid notch: the natural history of infraspinatus atrophy in volleyball players. The American Journal of Sports Medicine, v.26, n.6, p.759-63.

7. Freedman L, Munro RR (1966). Abduction of the arm in the scapular plane: scapular and glenohumeral movement. Journal of Bone and Joint Surgery [Am], v.48, n.8, p.1503-10.

8. Gerber C, Krushell RJ (1991). Isolated rupture of the tendon of the subscapularis muscle. Journal of Bone and Joint Surgery [Br], v.73, n.3, p.389-94.

9. Glousman R, Jobe FW, Tibone J, Moynes D, Antonelli D, Perry J (1988). Dynamic electromyografic analysis of the throwing shoulder with glenohumeral instability. Journal of Bone and Joint Surgery [Am], v.70, n.2, p.220-8.

10. Gowan ID, Jobe FW, Tibone JE, Perry J, Moynes DR (1987). A comparative electromyographic analysis of the shoulder during pitching: professional versus amateur pitchers. The American Journal of Sports Medicine, v.15, n.6, p.586-90.

11. Gross ML, Brenner SL, Esformes I, Sonzogni JJ (1993). Anterior shoulder instability in weight lifters. The American Journal of Sports Medicine, v.21, n.4, p.599-603.

12. Hovelius L, Thorling J, Fredin H (1979). Recurrent anterior dislocation of the shoulder: results after the Bankart and Putti-Platt operations. Journal of Bone and Joint Surgery $[\mathrm{Am}], \mathrm{v} .61, \mathrm{n} .4, \mathrm{p} .566-9$.

13. Howell SM, Imobersteg AM, Seger DH, Marone PJ (1986). Clarification of the role of the supraspinatus muscle in shoulder function. Journal of Bone and Joint Surgery [Am], v.68, n.3, p.398-404.

14. Jobe FW, Tibone J.E, Perry J, Moynes DR (1983).An EMG analysis of the shoulder in pitching: a preliminary report. The American Journal of Sports Medicine, v.12, n.3, p.3-5.

15. Jobe FW, Moynes DR, Tibone JE, Perry J (1984). An EMG analysis of the shoulder in pitching: a second report. The American Journal of Sports Medicine, v.12, n.3, p.218-20.

16. Jobe FW, Bradley JP (1987). Rotator cuff injuries in baseball: prevention and rehabilitation. Sports Medicine, v.6, p.378-87.

17. Kretzer HH, Richardson AB (1989). Rupture of the pectoralis major muscle. The American Journal of Sports Medicine, v.17, n.4, p.453-8.

18. Kumar VP, Balasubramanian P (1985). The role of atmospheric pressure in stabilizing the shoulder. Journal of Bone and Joint Surgery $[\mathrm{Br}], \mathrm{v} .67, \mathrm{n} .1, \mathrm{p} .719-21$.
19. Mcmaster WC, Long SC, Caiozzo VJ (1991). Isokinetic torque imbalances in the rotator cuff of the elite water polo player. The American Journal of Sports Medicine, v.19, n.1, p.72-5.

20. Milgrom C, Schaffler M, Gilbert S, Van Holsbeek M (1995). Rotator cuff changes in asymptomatic adults: the effect of age, hand dominance and gender. Journal of Bone and Joint Surgery [Br], v.77, n.2, p.296-8.

21. Neer CS (1972). Anterior acromioplasty for the chronic impingement syndrome in the shoulder. Journal of Bone and Joint Surgery $[\mathrm{Am}], \mathrm{v} .54, \mathrm{n} .1, \mathrm{p} .41-50$,

22. Nuber GW, Jobe FW, Perry J, Moynes DR, Antonelli D. (1986) A fine wire electromyography analysis of muscles of the shoulder during swimming. The American Journal of Sports Medicine, v.14, n.1, p.7-11.

23. O'Brien, S. J.; Neves MC, Arnoczky SP, Rozbruck SR, Dicarlo EF, Warren RF, Schwartz R, Wickiewicz TL. (1990). The anatomy and histology of the inferior glenoumeral ligament complex of the shoulder. The American Journal of Sports Medicine, v.18, n.5, p.449-56.

24. O'Connell PW, Nuber GW, Mileski RA, Lautenschlager RE (1991). The contribution of the glenoumeral ligaments to anterior stability of the shoulder joint. The American Journal of Sports Medicine, v.19, n.6, p.579-84.

25. Oestern HJ, Blankenburg PV, Defino HLA (1993). Estudo morfológico e biomecânico dos tendões do músculo supraespinhoso e porção longa do bíceps braquial. Revista Brasileira de Ortopedia, v.28, n.11, p.843-5.

26. Pappas MA, Zawacki RM, Sullivan TJ (1985). Biomechanics of baseball pitching: a preliminary report. The American Journal of Sports Medicine, v.13, n.4, p.216-22

27. Pecina MM, Krmpotic-nemanic J, Markiewitz AD (1991). Tunnel syndromes. Boca Raton, CRC, p.23-5.

28. Pecina MM, Bojanic D (1993). Overuse injuries of the musculoskeletal system. Boca Raton, CRC, p. 31-54.

29. Pelham TW, Holt LE, Stalker RE (1995). The etiology of paddler's shoulder. The Australian Journal of Science and Medicine in Sport, v.27, n.2, p.43-7.

30. Pink M, Perry J, Browne A, Scovazzo ML, Kerrigan J (1991). The normal shoulder during freestyle swimming: an electromyographic and cinematographic analysis of twelve muscles. The American Journal of Sports Medicine, v.19, n.6, p.569-76.

31. Poppen NK, Walker PS (1976). Normal and abnormal motion of the shoulder. Journal of Bone and Joint Surgery [Am], v.58, n.2, p.195-201.

32. Powers ME (1998). Rotator cuff training for pitchers. Journal of Sport Rehabilitation, n.7, p.285-99.

33. Radin EL (1993). Mechanically induced periarticular and neuromuscular problems. In: Wright V, Radin EL. Mechanics of human joints: physiology, pathophysiology and treatment. New York, Marcel Dekker, p.355-70.

34. Rathbun JB, Macnab L (1970). The microvascular pattern of the rotator cuff. Journal of Bone and Joint Surgery $[\mathrm{Br}]$, v.52, n.3, p.540-53.

35. Richardson AB, Jobe FW, Collins HR (1980). The shoulder in competitive swimming. The American Journal of Sports Medicine, v.19, n.6, p.159-63.

36. Rothstein JM, Lamb RL, Mayhew TP (1987). Clinical uses of isokinetic measurements. Physical Therapy, v.67, n.12, p.1840-4. 
37. Ryu RKN, Mccormick J, Jobe FW, Moynes DR, Antonelli DJ (1988). An electromyographic analysis of shoulder function in tennis players. The American Journal of Sports Medicine, v.16, n.5, p.481 -5.

38. Saidoff DC, Mcdonough AL(1997). Critical pathways in therapeutic intervention: upper extremity.St. Louis, Mosby.

39. Silver RL, La Garza J, Rang M (1985). The myth of muscle balance. Journal of Bone and Joint Surgery [Br], v.67, n.3, p.432-7.

40. Symeonides PP (1972). The significance of the subescapularis muscle in the pathogenesis of recurrent anterior dislocation of the shoulder. Journal of Bone and Joint Surgery $[B r], v .54$, n.3, p.476-83.

41. Terry GC, Hammon D, France P, Norwood LA (1991). The stabilizing function of passive shoulder restraints. The American Journal of Sports Medicine, v.19, n.1, p.26-34.

42. Towsend H, Jobe FW, Pink M, Perry J (1991).

Electromyografic analysis of the glenoumeral muscles during a baseball rehabilitation program. The American Journal of Sports Medicine, v.19, n.3, p.264-72.

43. Van der Helm FCT (1994). Analysis of the kinematic and dynamic behavior of the shoulder mechanism. Journal of Biomechanics, v.27, n.5, p.527-50

44. Van Linge B, Mulder JD (1963). Function of the supraspinatus muscleand its relation to the supraspinatus syndrome: an experimental study in man. The Journal of Bone and Joint Surgery $[\mathrm{Am}]$, v.45, n.4, p.750-754.

45. Warner JJP, Deng X, Warren RF, Torzilli PA (1992). Static capsuloligamentous restraints to superior-inferior translation of the glenoumeral joint. The American Journal of Sports Medicine, v.20, n.6, p.675-85.

46. Zoppi Filho A. Envelhecimento biológico do ombro: estudo em 40 ombros dissecados. São Paulo, 1988. 89p. Dissertação (Mestrado) - Faculdade de Medicina, Universidade de São Paulo. 